\title{
Fiber optic displacement measurement model based on finite reflective surface
}

\author{
YUHE LI*, KAISEN GUAN, ZhAOHUI HU
}

\author{
The State Key Laboratory of Precision Measuring Technology and Instruments, Precision Instrument Department, Tsinghua University, Beijing \\ 100084, China \\ *Corresponding author: liyuhe@mail.tsinghua.edu.cn
}

\begin{abstract}
We present a fiber optic displacement measurement model based on finite reflective plate. The theoretical model was derived, and simulation analysis of light intensity distribution, reflective plate width, and the distance between fiber probe and reflective plate were conducted in details. The three dimensional received light intensity distribution and the characteristic curve of light intensity were studied as functions of displacement of finite reflective plate. Experiments were carried out to verify the established model. The physical fundamentals and the effect of operating parameters on measuring system performance were revealed in the end.
\end{abstract}

Keywords: Displacement measurement; Fiber optic sensing; Numerical approximation and analysis.

\section{Introduction}

Displacement measurement sensors are the core components of advanced instruments. The capacitive displacement sensor is a kind of non-contact sensor and has ultra-high-resolution down to $0.01 \mathrm{~nm}$ [1]. However, it has a limited measurement range and high cost while it is hard to meet its high-level application requirements. The inductive displacement sensor has long measuring range with accuracy up to 0.1 $\mu \mathrm{m}$, and its dynamic characteristics are not so high comparatively [2]. Laser interference displacement sensor has large measurement range and high accuracy of nanometer level, as well as the ability of measuring linear and angular displacement at the same time. However it is too complex and expensive and it would be difficult to meet stringent environmental requirements [3]. Grating displacement sensor utilizes moire fringes to measure in-plane displacement with large measuring range and high accuracy of $5 \mu \mathrm{m}$ [4]. By contrast, both dial gauge and encoder are medium accuracy, low-cost and widely applied.

Recently fiber optic sensors are applied in many kinds of measurement and control systems, such as industry, medicine, military, aerospace, etc. Fiber optic has many advantages of extremely simple structure, low fabrication cost, high sensitivity, high resistance to electromagnetic interference, and it is suitable for many harsh environments [5,6]. The reflective optical fiber sensors utilize modulation of reflected light intensity to measure displacement [7-10], which have very simple structure and large measurement range. An inclined two fiber displacement sensor is also presented [11], with the dependences of performance metrics reported. Another kind of optical fiber sensor makes use of intensity modulation of transmitted light [12]. Fiber Bragg grating based sensors, which adopt wavelength modulation, are widely used for displacement measurement $[13,14]$. Fiber optic Sagnac interferometer uses light phase modulation to measure displacement, but its structure is complex [15]. Nowadays great attention is focused on the fiber optic displacement sensors (FODS) based on reflected light modulation.

It is important to establish and analyze the theoretical model of reflective fiber optic sensors to provide theoretical basis for design. Most existing reflective fiber optic sensors are based on large enough reflective planes, especially in fiber optic distance measurement sensors [9-11]. Furthermore, there are also needs for fiber optic sensors with finite reflective surface to measure displacement. A benttip optical fiber sensor based on finite reflective surface is developed for soft tissue investigation during minimally invasive surgery [16]. The sensor measures the shift of the finite flat reflector to sense the force, which investigate mechanical soft tissue properties. And a reflective intensity modulated optical fiber sensor is presented to measure rotation axis transverse displacements [17], which also utilizes finite reflector. However, existing fiber optic displacement measurement models have many approximate conditions, and most of them only analyze the size of received light intensity instead of the three-dimensional distribution.

The accurate analysis of reflective fiber optic sensors based on finite reflective surface is theoretical basis for the design and establishment of sensors and systems, in which the reflective surface needs to be finite. The theoretical analysis is helpful to fix the parameters of fiber optics, which improves the performance of fiber optics displacement measurement sensors. Therefore a fiber optic displacement measurement model based on finite reflective surface is proposed in this work. The mathematical model is setup while the theoretical analysis and simulation with various illustrative examples for the corresponding model are performed. The three dimensional light intensity distribution and the characteristic curve of light intensity were studied as functions of displacement of finite reflective plate. Experiments are carried out to verify the established model. Further analysis of light intensity modulation, which will change with the measurement system parameters, is conducted at the end.

\section{Theoretical Model}

\subsection{Measuring Principle}

The measuring principle is shown in Fig. 1. The measurement system includes some important components, such as light source, transmitting fiber, receiving fiber, finite reflective plate, and optical power meter. The transmitting and receiving fibers constitute a fiber optic probe, with a certain angle between every fiber and the probe's central axis. The finite reflective plate is a long strip, which is long 
enough in its length direction and finite in width direction with respect to the diameter of optical fiber. The light is emitted for the source and transmitted into the transmitting fiber. After emitted from the fiber end, the light is reflected by the finite reflective plate and coupled into the receiving fiber. Then the reflected light intensity is detected by the optical power meter. When the finite reflective surface moves in the vertical and lateral directions, the receiving light intensity will be modulated and change accordingly, which can be used for measuring distance and displacement. In this work, displacement refers to the lateral movement of the reflective surface, and distance is the longitudinal movement between the fibers and the reflective surface.

Normally, light exits an end-emitter fiber in a hypothetical cone of light defined by the numerical aperture (NA) of the fiber, and every light point on the end face of transmitting fiber brings about a local light cone which would be combined to form the whole emitting light [18]. The light intensity distribution on the end face of transmitting fiber is considered as Gauss distribution, as well as the light intensity distribution on every section of local light cone. It is supposed that the light travels in straight lines and specular reflection occurs on the reflective surface. Only a part of light is reflected into the receiving fiber and transformed in electric signal.

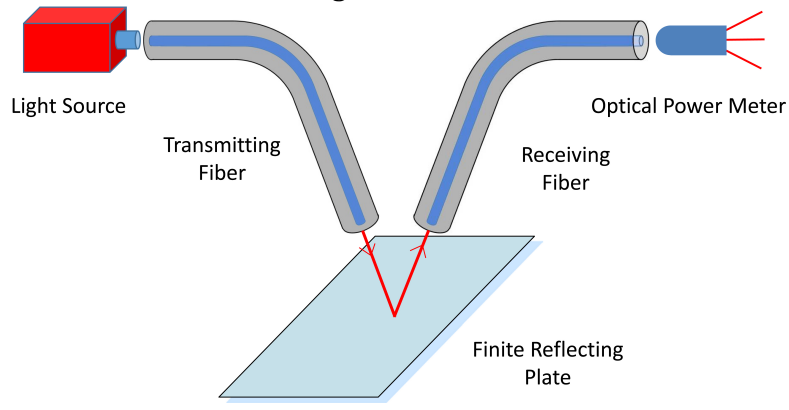

Fig. 1. Principle of measurement system.

\subsection{Mathematical Modeling}

The fiber optic sensing system is composed of a fiber optic probe and a rectangular finite reflective plate as shown in Fig. 2(a), and the transmitting fiber $\Phi$ and receiving fiber $\Psi$ constitute a fiber optic probe. As shown in Fig. 2(b), the angle between fibers and vertical axis is $\alpha$, and the horizontal distance between the center points $O_{\mathrm{T}}$ and $O_{\mathrm{R}}$ on the end face of fibers is $S$. The core radii of fibers with same numerical aperture NA, are $R_{t}$ and $R_{r}$ respectively. The reflective plate $\mathrm{P}$ has finite size in width direction and rectangular shape, and is placed horizontally below the fiber optic probe. The width direction is parallel to the plane formed by the center axes of fibers. Due to the finite width $l$

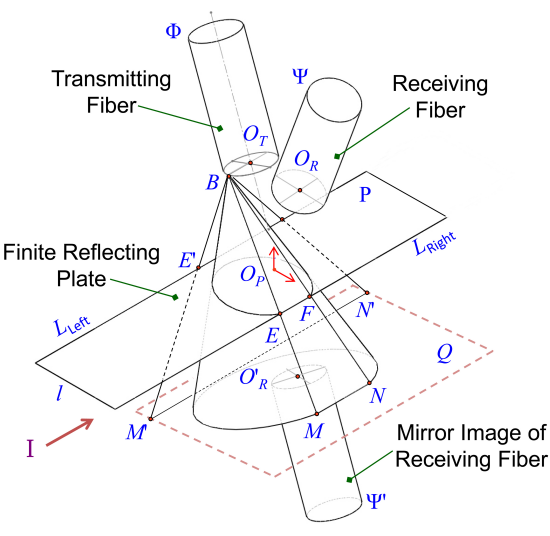

(a)

$$
\text { ) }
$$

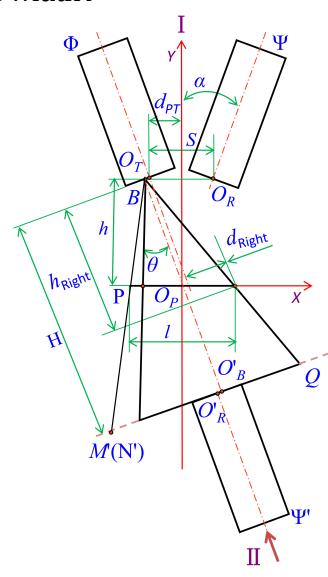

(b) of reflecting plate, it may occur that a certain amount of transmitting light cannot be reflected back into the receiving fiber. The distance between the reflective plate $P$ and the center point $O_{\mathrm{T}}$ on the end face of fiber is $h$, and the distance between the center line along the length direction of reflective plate $\mathrm{P}$ and the center point $O$ is $d_{P T}$.

In this model, the cone angle of transmitted light $\theta$ is determined by NA, $\theta=\arcsin (\mathrm{NA})$. The end face of transmitting fiber $\Phi$ can be denoted as $\odot O_{\mathrm{T}}$. Every point on $\odot O_{\mathrm{T}}$ forms a normal light cone, and all light emitted from the emitting point is within the normal cone [18]. The light intensity distribution on the plane of receiving fiber $\Psi$ is a linear superposition of all light cones reflected on the finite plate $\mathrm{P}$ from all emitting points on the end face of transmitting fiber $\Phi$. The theoretical light intensity which is the most important physical parameter for fiber optic sensing, can be calculated from the mathematical model.

Based on the law of reflection the reflected light is coupled into the receiving fiber $\Psi$. Here the mirror image $\Psi$ ' of receiving fiber is used to substitute for the receiving fiber $\Psi$, so the light reflection can be transformed into the linear transmission of light, which is easier for the further processing of mathematical model. The infinite plane passing through the end face of the mirror image $\Psi$ ', is labeled as $Q$.

The flow chart of the whole mathematical calculation is shown in Fig. 3. Specific calculation process is presented as follows.

Step 1, establish two coordinate systems, as shown in the Fig. 2(b),(c). $O_{\mathrm{T}}, O_{\mathrm{R}}$ and $O_{\mathrm{P}}$ are the centers of the end faces of fibers and the reflective plate $\mathrm{P}$, respectively. In the front view there is a coordinate system named $X O_{\mathrm{P}} Y$, with origin $O_{\mathrm{P}}, X$ axis along the width direction of plate $\mathrm{P}$ and $Y$ axis in the vertical direction of plate $\mathrm{P}$. In the perspective view on the plane opposite the end face of the receiving fiber, set another coordinate system named $x O_{\mathrm{T} y}$, with origin $O_{\mathrm{T}} x$ axis in the width direction of plate $\mathrm{P}, y$ axis being parallel to plate $\mathrm{P}$.

Step 2, choose a point B randomly as an initialized point.

Step 3, calculate its light intensity. Choose a random emitting point B on the end face of the transmitting fiber, whose coordinates are $\left(x_{\mathrm{B}}, y_{\mathrm{B}}\right)$. The light intensity follows a Gaussian distribution, consequently the light intensity at point $B$ can be calculated as follows:

$$
I\left(x_{B}, y_{B}\right)=\frac{2 \eta P_{0}}{\pi \omega_{0}^{2}} \exp \left[-\frac{2\left(x_{B}^{2}+y_{B}^{2}\right)}{\omega_{0}^{2}}\right],
$$

where $P_{0}$ is the power of light source, $\eta$ is the transmission efficiency of transmitting fiber, $\omega_{0}$ is the Gauss width at 1/e peak value. Because the distance between the transmitting fiber and the mirror image of receiving fiber is smaller than Rayleigh Range, we set Gauss width equal to the radius of fiber, namely $\omega_{0}=R_{t}[10]$.

Fig. 2. (a) Mathematical model of measurement system. (b) Mathematical model variables. (c) Geometric projection.

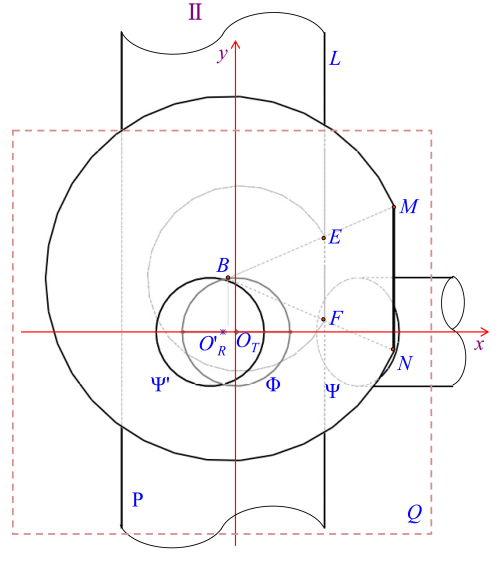

(c) 


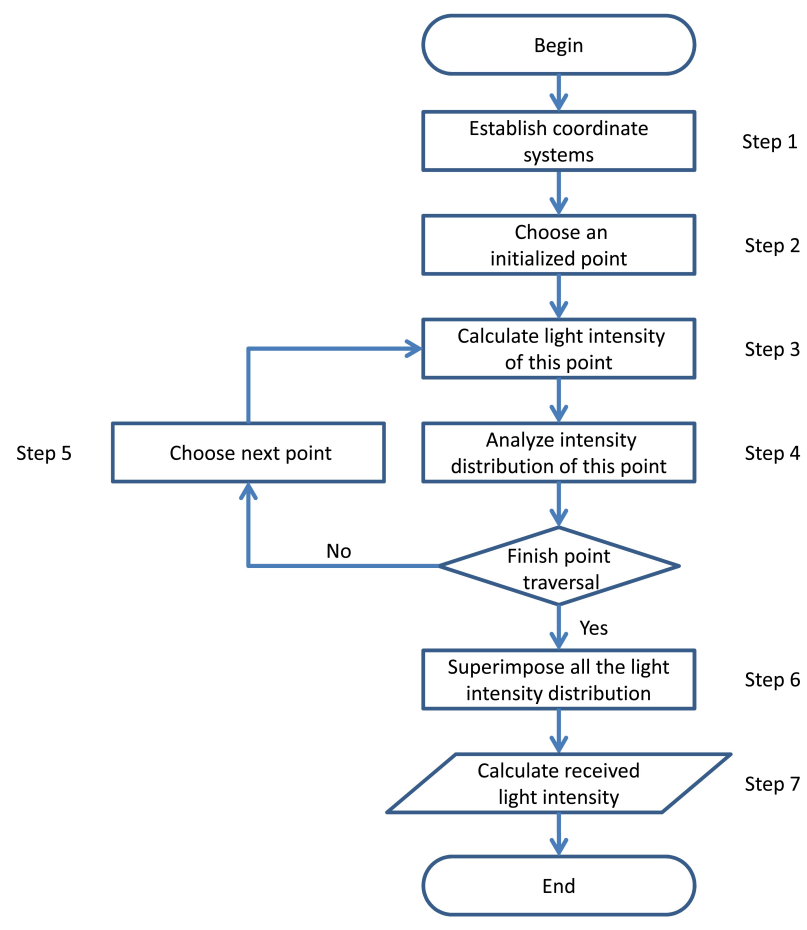

Fig. 3. Flow chart of calculation process

Step 4, analyze the intensity distribution of point $B$ formed on the end face of receiving fiber.

Step 4.1, due to the finite reflective surface of finite width and infinite length, one part of the light cone from point $B$ reaches the reflective plate and is reflected into the end face of the receiving fiber, while the other part cannot reach the end face of fiber, therefore it doesn't participate in the calculation of light intensity distribution (LID).

Step 4.2, calculate the region $R_{\mathrm{B}-Q}$ of the light spot on the plate $Q$, which is formed by the light cone from the emitting point $B$. The light spot should fall within the circle $\odot O_{B}^{\prime}$, with center $O_{B}^{\prime}$, and radius $R_{B}=H \cdot \tan \theta$. Here $H$ is the distance between the end face of transmitting fiber and the plane $Q$. Furthermore, the contour of reflected light spot is affected by the shape and size of the finite reflective plate P. In some case, as shown in Fig. 2(a), the right edge of plate $\mathrm{P}$ would alter the shape of light spot. The region $O_{\mathrm{p}} E F$ formed by the light cone and the reflector $\mathrm{P}$, would in general change with the width and position of reflective plate $\mathrm{P}$ accordingly, and it divides the light cone into two parts, and only the internal part reaches the receiving fiber by reflector P. As shown in Figs. 2(a) and 2(c), the plane BEF and infinite plane $Q$ intersect at a line $M N$, then the reflected light can only reach the inner side of $M N$ which would be derived as follows.

As shown in Fig. 2(b), the distance $h_{\text {Right }}$ between the reflector's right side $L$ right and the plane of $\odot O_{\mathrm{T}}$, equals to the distance between the two points $E(l / 2,0)$ and $O_{\mathrm{T}}\left(d_{P T}, h\right)$ along the length direction of transmitting fiber $\Phi$, which can be expressed as following:

$$
h_{\text {Right }}=(l \sin \alpha) / 2-d_{P T} \sin \alpha+h \cos \alpha .
$$

And the distance between the right side $L_{\text {right }}$ and the center axis of transmitting fiber $\Phi$ is denoted as $d_{\text {Right: }}$

$$
d_{\text {Right }}=(l \cos \alpha) / 2-d_{P T} \cos \alpha-h \sin \alpha .
$$

Similarly, we can get the distances $h_{\text {Left }}$ and $d_{\text {Left }}$ from the reflector's left side $L_{\text {Left }}$ as follows:

$$
\left\{\begin{array}{l}
h_{\text {Left }}=-(l \sin \alpha) / 2-d_{P T} \sin \alpha+h \cos \alpha . \\
d_{\text {Left }}=-(l \cos \alpha) / 2-d_{P T} \cos \alpha-h \sin \alpha
\end{array}\right.
$$

The distance between the two planes of $\odot O_{\mathrm{T}}$ and $\odot O_{\mathrm{R}}^{\prime}$ is:

$$
H=S \sin \alpha+2 h \cos \alpha .
$$

As shown in Fig. 2(c), the lines of $M^{\prime} N^{\prime}$ and $M N$ are the left and right borders of the region of light spot on the plate $Q$, formed by the left and right edges $L_{\text {Left }}$ and $L_{\text {right }}$ of the reflector P. The positions of the two borders in coordinate system $x O_{\mathrm{T}} y$ can be calculated using the formulas below.

$$
\left\{\begin{array}{l}
x_{M^{\prime} N^{\prime}}=H\left(d_{\text {Left }}-x_{B}\right) / h_{\text {Left }}+x_{B} \\
x_{M N}=H\left(d_{\text {Right }}-x_{B}\right) / h_{\text {Right }}+x_{B} .
\end{array}\right.
$$

Therefore, an arbitrary point $\left(x_{r}, y_{r}\right)$ on the light spot should meet the condition:

$$
X_{M^{\prime} N^{\prime}} \leq X_{r} \leq X_{M N} .
$$

Consequently, the region $R_{\mathrm{B}-\mathrm{Q}}\left(\mathrm{x}_{r}, y_{r}\right)$ of the light spot from point $B$ reflected by infinite plate $\mathrm{P}$ is calculated as follows:

$$
R_{B-Q}\left(x_{r}, y_{r}\right)=\left\{\begin{array}{l}
\left(\left[\left(x_{r}-x_{B}\right)^{2}+\left(y_{r}-y_{B}\right)^{2}\right] \leq(H \tan \theta)^{2}\right) \\
\&\left(x_{r} \geq H\left(d_{\text {Left }}-x_{B}\right) / h_{\text {Left }}+x_{B}\right) \\
\&\left(x_{r} \leq H\left(d_{\text {Right }}-x_{B}\right) / h_{\text {Right }}+x_{B}\right)
\end{array}\right\} .
$$

Step 4.3, calculate the LID formed by point $B$. The LID of every section of the light cone emitted from point can be approximated by a Gaussian distribution. Therefore, in coordinate system $x O_{\mathrm{T}} y$ the LID on the plane of the end face of receiving fiber $\Psi$, formed by the light cone from emitting point $B$, is given as follows:

$$
\Delta I_{r}\left(x_{r}, y_{r}\right)=\frac{2 \Delta P_{B}}{\pi \omega^{2}(H)} \exp \left\{-\frac{2\left[\left(x_{r}-x_{B}\right)^{2}+\left(y_{r}-x_{B}\right)^{2}\right]}{\omega^{2}(H)}\right\} \square\left(x_{r}, y_{r}, x_{B}, y_{B}\right)
$$

where,

$$
\begin{aligned}
& \Delta P_{B}=I\left(x_{B}, y_{B}\right) \Delta x_{B} \Delta y_{B}=\frac{2 \eta P_{0}}{\pi \omega_{0}{ }^{2}} \exp \left[-\frac{2\left(x_{B}{ }^{2}+y_{B}{ }^{2}\right)}{\omega_{0}{ }^{2}}\right] \Delta x_{B} \Delta y_{B}, \\
& \omega(H)=H \tan (\arcsin (N A)), \\
& f\left(x_{r}, y_{r}, x_{B}, y_{B}\right)= \begin{cases}1, & \left(x_{r}, y_{r}\right) \subseteq R_{B-Q} . \\
0, & \text { Other }\end{cases}
\end{aligned}
$$

Here $\left(x_{r}, y_{r}\right)$ describes an arbitrary point on the plane of the end face of the receiving fiber in $x O_{\mathrm{T}} y I\left(x_{B}, y_{B}\right)$ is the light intensity of point $B . \Delta x_{B}$ and $\triangle y_{B}$ represent the micro distance values along $x$ and $y$ axes, respectively. $\Delta P_{B}$ means the total power of point $B$, which is a micro quantity. $\omega(H)$ is the Gauss width of the light intensity distribution, and $f\left(x_{r}, y_{r}, x_{B}, y_{B}\right)$ is a Boolean function.

Step 5, choose next point on the end face of the transmitting fiber and go to step 2, until all the points have been calculated.

Choose a new point on the end face and conduct step 2 and step 3 repeatedly. Then the LID of every point on the end face of transmitting fiber is calculated.

Step 6, superimpose all the light intensity distribution to obtain the whole distribution on the plane of the end face of the receiving fiber.

The whole and complete LID on the plane of the end face of receiving fiber is a linear superimposition. It is shown discretely as follows:

$$
\begin{aligned}
& I_{r}\left(x_{r}, y_{r}\right)=\sum_{\left.\left(x_{r}^{2}+r_{r}\right)^{2}\right) R_{r}} \frac{2 \Delta P_{t}}{2 \pi \omega^{2}(H)} \exp \left\{-\frac{2\left[\left(x_{r}-x_{t}\right)^{2}+\left(y_{r}-x_{t}\right)^{2}\right]}{\omega^{2}(H)}\right]\left[f\left(x_{r}, y_{r}, x_{t}, y_{t}\right)\right. \\
& =\sum_{\left(x_{t}^{2}+t_{t}^{2}\right)<R_{t}^{2}} \frac{2}{\pi \omega^{2}(H)} \exp \left\{-\frac{2\left[\left(x_{r}-x_{t}\right)^{2}+\left(y_{r}-x_{t}\right)^{2}\right]}{\omega^{2}(H)}\right\} \frac{2 \eta P_{0}}{\pi \omega_{0}^{2}} \exp \left[-\frac{2\left(x_{t}^{2}+y_{t}^{2}\right)}{\omega_{0}^{2}}\right] f f\left(x_{t}, y_{r}, x_{t}, y_{t}\right) \Delta x_{t} \Delta y_{t}
\end{aligned}
$$

It can be rewritten in integration:

$I_{r}\left(x_{r}, y_{r}\right)=\iiint_{D\left(x_{t}^{2}+y_{t}^{2}\right)<R_{t}^{2}} \sum \frac{2}{\pi \omega^{2}(H)} \exp \left\{-\frac{2\left[\left(x_{r}-x_{t}\right)^{2}+\left(y_{r}-x_{t}\right)^{2}\right]}{\omega^{2}(H)}\right] \frac{2 \eta P_{0}}{\pi \omega_{0}^{2}} \exp \left[-\frac{2\left(x_{t}^{2}+y_{t}^{2}\right)}{\omega_{0}^{2}}\right] f\left(x_{r}, y_{r}, x_{t}, y_{t}\right) d x_{t} d y_{t}$ 
Step 7, calculate the received light intensity of receiving fiber. Combined with the location of the receiving fiber and its radius, the received light intensity of receiving fiber can be calculated. In coordinate system $x O_{\mathrm{T}} y$ the distance between the center of transmitting and receiving fibers is $s=S \cos \alpha$ - $2 h \sin \alpha$, consequently the total received power of receiving fiber can be expressed as follows:

$$
P_{r}\left(h, S, l, d s, R_{t}, R_{r}, N A\right)=\iint_{(x-s)^{2}+y^{2} \leq R_{r}^{2}} I_{r}(x, y) d x d y
$$

\section{Model Simulation and Analysis}

\subsection{Model Simulation}

Computer simulation was carried out to check the consistency of theoretical model. Suppose that $R_{r}=25 \mu \mathrm{m}, R_{t}=25 \mu \mathrm{m}, h=100 \mu \mathrm{m}$, $l=100 \mu \mathrm{m}, S=60 \mu \mathrm{m}, \alpha=20^{\circ}, \mathrm{NA}=0.22, \eta=1$ and $P_{0}=1 \mathrm{nW}$. As shows in Fig. 4 , the finite reflective plane $P$ is moved from infinity toward the receiving fiber $\Psi$, and then moving away again toward infinity. In the process described above the value of $d_{P T}$ is changed from negative infinity to positive infinity. When the absolute value of $d_{P T}$ is large enough, the received light power would be theoretically equal to zero. Accordingly, when the absolute value is relatively small, some light reflected by finite plate $P$ would be emitted into the receiving fiber $\Psi$ and the received light power is greater than zero.

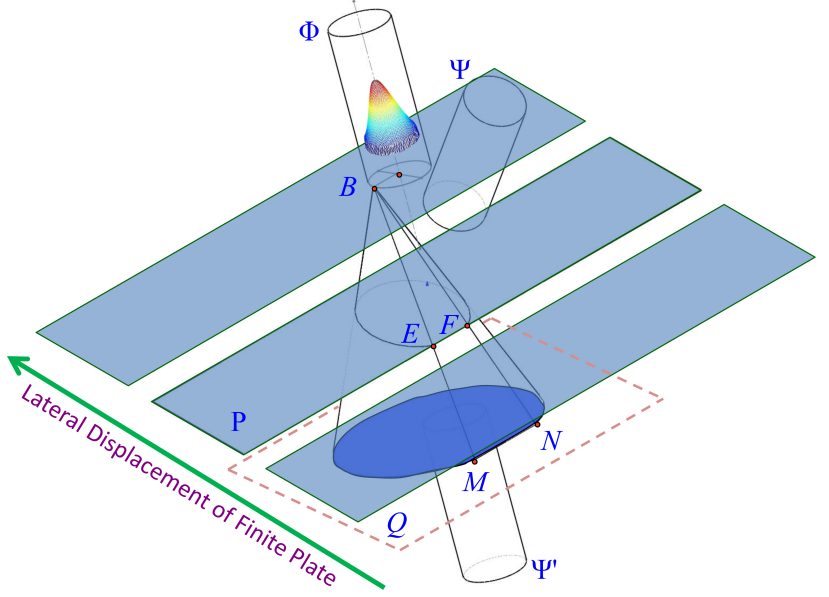

Fig. 4. Model simulation.

With the displacement of plate $\mathrm{P}$, which is represent by the change of $d_{P T}$ from negative to positive infinity, the LID on the plane of receiving fiber's end face and that received by receiving fiber would change synchronously, as shown in Fig. 5. The unit of $x$ axis and $y$ axis is $\mu \mathrm{m}$ while the unit of $z$ axis is $\mathrm{nW} /(\mu \mathrm{m})^{2}$. At the beginning of above process, the LID on the plane of receiving fiber's end face equals zero, and then the light intensity becomes larger and the range of the LID expands until the light intensity falls back to zero again in the end. As for the settled location of receiving fiber in coordinate system $x O_{\mathrm{T}} y$, the received LID of receiving fiber can be obtained from the region of the receiving fiber on the plane of receiving fiber's end face. Take the integral of the LID to get the received light intensity. In the end the curve of the received light intensity versus the displacement of finite plate $P$, is plotted in Fig. 6. The plate is moved from $-140 \mu \mathrm{m}$ to $50 \mu \mathrm{m}$ and the light intensity is from zero to $0.128 \mathrm{nW}$.
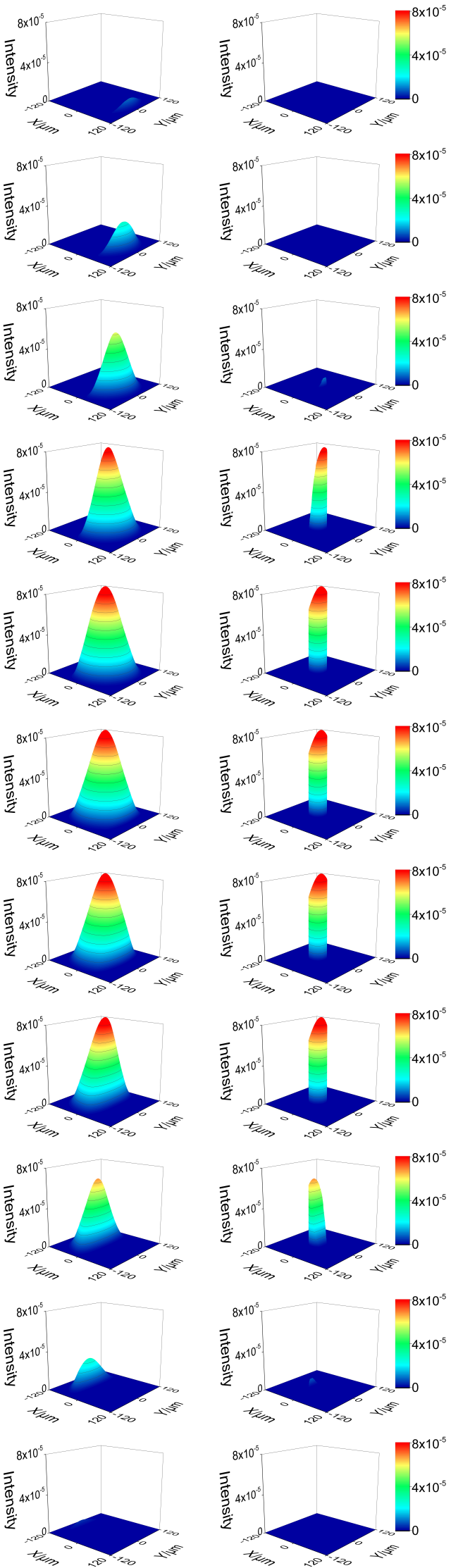

Fig. 5. Comparisons of the LID between the plane of end face (left) and receiving fiber (right) vs the displacement of plate $P$. 


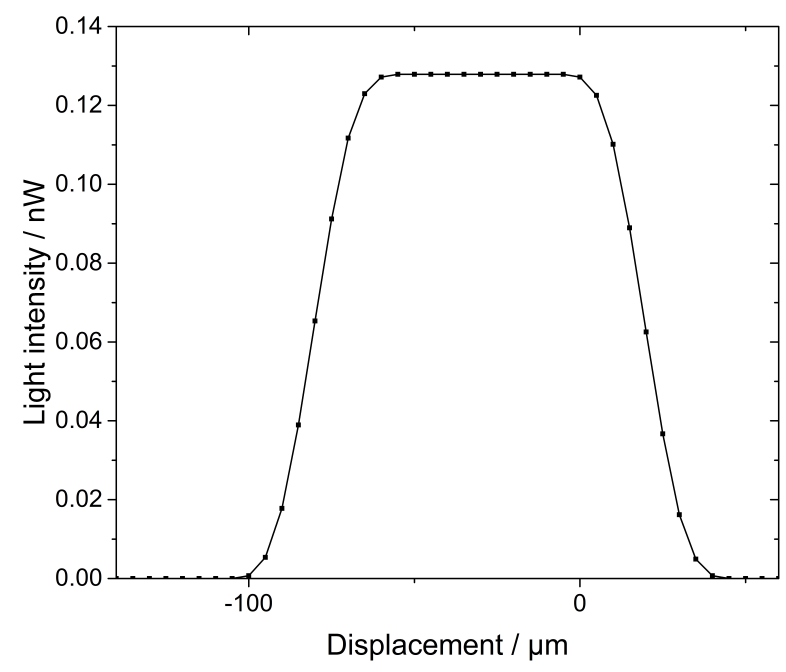

Fig. 6. Characteristic curve of light intensity versus displacement.

\subsection{Simulation Discussions}

Change the values of some parameters, such as the finite width $l$ of reflecting plate, the distance $h$ between reflective plate and the center point $O_{\mathrm{T}}$, and NA of the optical fibers, to discuss the characteristics of displacement measurement under the simulated conditions that the reflective plate moves laterally.

\subsubsection{Change Width I}

Simulation conditions: $R_{r}=25 \mu \mathrm{m}, R_{t}=25 \mu \mathrm{m}, h=600 \mu \mathrm{m}, S=125 \mu \mathrm{m}$, $\alpha=0, \mathrm{NA}=0.22, \eta=1, P_{0}=1 \mathrm{nW}$, and $l$ changing from $50 \mu \mathrm{m}$ to $400 \mu \mathrm{m}$ with step $50 \mu \mathrm{m}$. The results were simulated and shown in Fig. 7, and the $x$ axis represents the displacement of the finite reflector $\mathrm{P}$, the $y$ axis being the normalized light intensity received by the receiving fiber $\Psi$. The plate is moved from $-400 \mu \mathrm{m}$ to $400 \mu \mathrm{m}$.

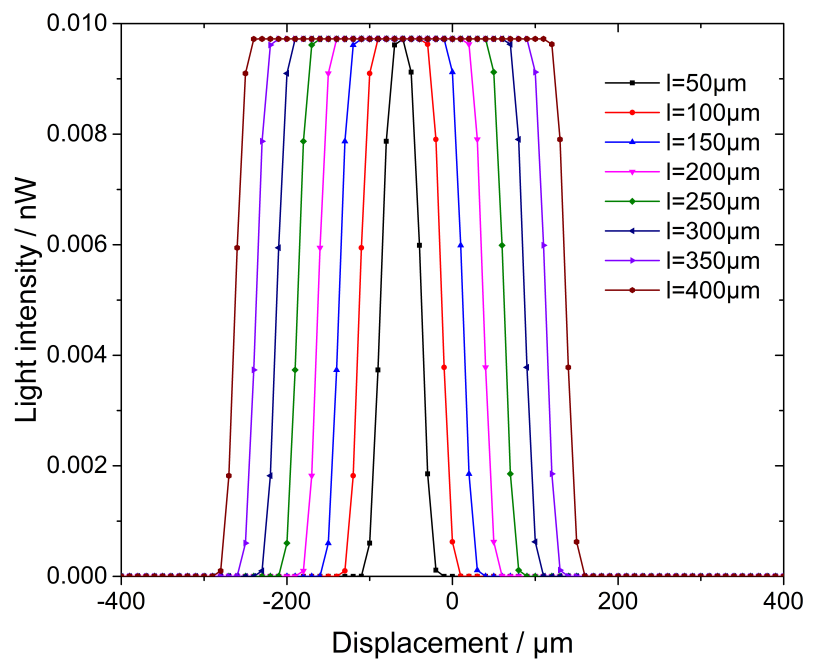

Fig. 7. Relationship between light intensity and displacement regarding width $l$.

As a whole, when the finite reflective plate is far away from the fiber optic probe, the received light intensity equals zero. While the reflector is close to the probe, the received intensity would grow to the maximum value, and then the intensity turns to zero again with the reflector far away from the probe. When the width $l$ is small enough, the top region of the curve would be retracted to a point. On the contrary the flat roof of curve comes out and becomes wider gradually with the increase of the width $l$. It is to say that as for small width $l$ the curve is close to a sine wave and a square wave for large width of finite reflective plate.

\subsubsection{Change Distance $h$}

Simulation conditions: $R_{r}=25 \mu \mathrm{m}, R_{t}=25 \mu \mathrm{m}, S=125 \mu \mathrm{m}, \alpha=0, l=300 \mu \mathrm{m}$ $\mathrm{NA}=0.22, \eta=1, P_{0}=1 \mathrm{nW}$, and $h$ changing from $100 \mu \mathrm{m}$ to $800 \mu \mathrm{m}$ with step $100 \mu \mathrm{m}$. The results were simulated and shown in Fig. 8 . The $x$ axis means the displacement of finite reflector $\mathrm{P}$, the $y$ axis means the distance $h$ while the $z$ axis means the normalized light intensity received by the receiving fiber $\Psi$. The plate is moved from $-400 \mu \mathrm{m}$ to $400 \mu \mathrm{m}$.

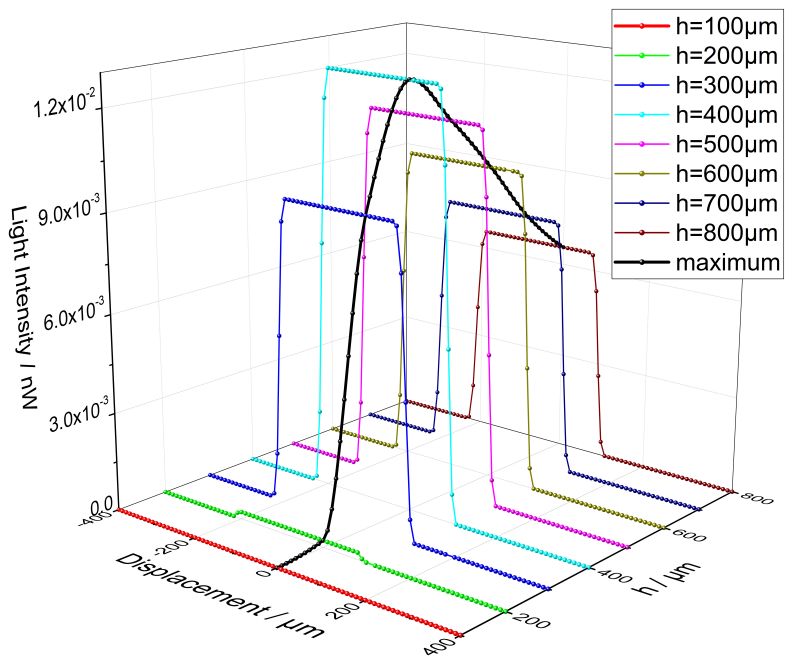

Fig. 8. Relationship between intensity and displacement regarding distance $h$.

From Fig. 8, it can be seen that every curve grows up from zero to its maximum, and then decreases to zero. With the increase of $h$, the maximum values of the normalized light intensity increase first and then decrease gradually. The curves exhibit a maximum with a steep linear front slope and back slope which can be practically used to measure displacement or distance based on fiber optic probe. Generally, the linear region of the front slope or back slope of each curve can be used to measure displacement. On one hand we choose appropriate distance $h$ and use the curve of the received light intensity versus the displacement of finite plate to measure displacement. On the other hand we fix the finite plate (displacement=0) and use "maximum curve" in Fig. 8 to measure distance, namely the longitudinal movement between the fibers and the reflective surface

\subsubsection{Change Numerical Aperture NA}

Simulation conditions: $R_{r}=25 \mu \mathrm{m}, R_{t}=25 \mu \mathrm{m}, h=600 \mu \mathrm{m}, S=125 \mu \mathrm{m}$, $\alpha=0, l=300 \mu \mathrm{m}, \eta=1, P_{0}=1 \mathrm{nW}$, and NA changing from 0.06 to 0.38 with step 0.04. The results were simulated and shown in Fig. 9. The $x$ axis means the displacement of finite reflector $\mathrm{P}$, the $y$ axis means the numerical aperture NA while the $z$ axis means the normalized light intensity received by the receiving fiber $\Psi$. The finite plate is moved from $-400 \mu \mathrm{m}$ to $400 \mu \mathrm{m}$. 


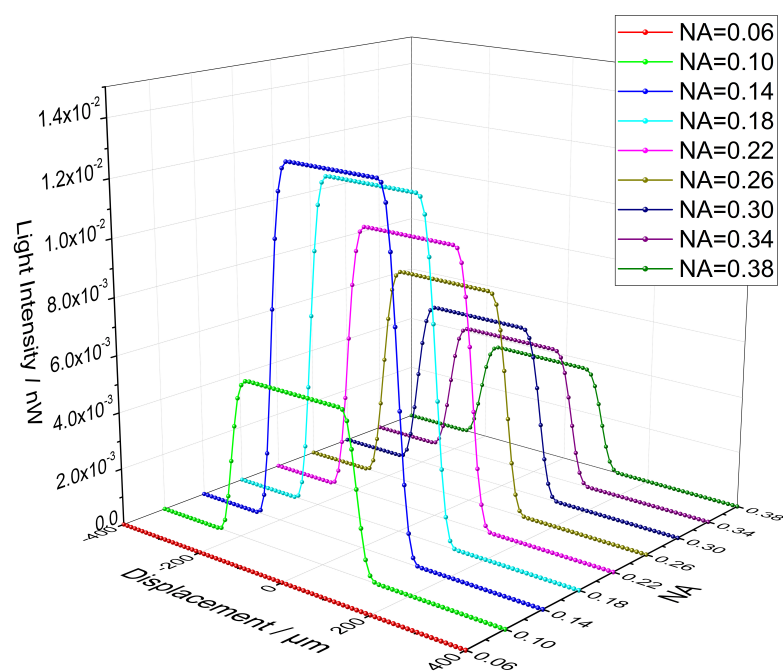

Fig. 9. Relationship between intensity and displacement regarding NA.

From Fig. 9, it can be seen that every curve grows up from zero to its maximum, and then decreases to zero, which is similar to the curves in Fig. 7 and Fig. 8. With the increase of $\mathrm{NA}$, the maximum values of the normalized light intensity increase first and then decrease gradually. NA has great influence on the linear scope of each curve, which determines the measuring range and resolution of lateral displacement measurement. Therefore, the simulations help us to choose fiber optics with appropriate NA for displacement measurement.

\section{Experiments and Model Verification}

\subsection{Level and Distribution of Received Light Intensity}

The experiment system, which includes the transmitting fiber, the receiving fiber and the finite reflective plate, is established, and some experiments are carried out. The beam profiler (Cinogy: CinCam CMOS-1202) was utilized to analyze the received light and to verify the received light intensity distribution obtained from the proposed theoretical model. The optical power meter (Thorlabs: PM320E) was used to measure the light intensity of receiving fiber and to be compared with theoretical curve. (a)

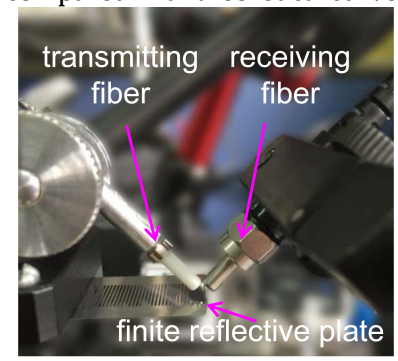

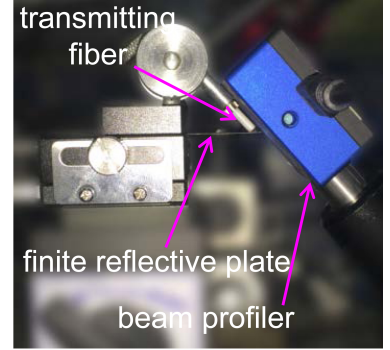

(b)
Fig. 10. Experimental setup. (a) transmitting and receiving fibers. (b) receiving fiber and beam profiler.
As shown in Fig. 10, the light beam is emitted from the transmitting fiber and then reflected by the finite reflective plate. After that the light is coupled into the receiving fiber and the received light intensity is measured by optical power meter. In addition, the beam profiler is placed on the plane of receiving fiber's end face to record the reflected LID on the end face.

Experimental conditions: $R_{r}=500 \mu \mathrm{m}, \quad R_{t}=25 \mu \mathrm{m}, \quad h=1100 \mu \mathrm{m}$, $l=300 \mu \mathrm{m}, S=2100 \mu \mathrm{m}, \alpha=45^{\circ}, \mathrm{NA}=0.22$. The transmitting fiber is multimode silica fiber with core radius of $25 \mu \mathrm{m}$ and cladding radius of $62.5 \mu \mathrm{m}$, while the receiving fiber is multimode plastic fiber with core radius of $500 \mu \mathrm{m}$ and cladding radius of $517.5 \mu \mathrm{m}$. The intensity profile of the optical spot can be regarded as Gaussian distribution and the speckle can be ignored [10]. The reflective surface is the polished steel. The reflector P moves horizontally and laterally, and the received light intensity and the distribution are obtained simultaneously. Then the experimental results are compared with the simulated result to verify the theoretical model.

In the upper half of Fig. 11, the black and red curves indicate theoretical and experimental light intensity, respectively. The $x$ axis is the displacement of the finite reflective plate. The $y$ axis of the red curve is the received light intensity from optical power meter, and the $y$ axis of the black curve is the normalized light intensity. The plate is moved from $-1700 \mu \mathrm{m}$ to $-600 \mu \mathrm{m}$. The two curves exhibit a remarkably high correlation. Overall as a whole relatively high agreement between theoretical and measured curves is obtained.

The theoretical and experimental LIDs are very similar, which are shown in the lower half of Fig. 11. With the horizontal displacement of finite reflector, the reflected LID would change gradually. The shape of distribution expands from a small spot and then disappears again, which is similar to a part of circle. In addition, most energy is concentrated at the center of the distribution. The total light power also increases from zero to maximum and then decreases to zero again. Based on a golden search optimization algorithm, which provided minimum RMS error between theoretical and measured values, we can get the RMS error $6.92 \%$. All the modeling results are coincidental with the experimental data.

\subsection{Influence of Width and Height on Fiber Optic Measurement}

\subsubsection{Influence of Width I}

The experiments are conducted by use of several finite reflective plates with different width. A series of curves are measured to verify the theoretical model and simulation analysis. Experimental conditions: $R_{r}=25 \mu \mathrm{m}, R_{t}=25 \mu \mathrm{m}, h=600 \mu \mathrm{m}, S=125 \mu \mathrm{m}, \alpha=0, \mathrm{NA}=0.22$, and $l$ changing from $100 \mu \mathrm{m}$ to $300 \mu \mathrm{m}$ with step of $100 \mu \mathrm{m}$.

The experimental results are shown in Fig. 12. The scatters are experimental result while the lines are simulation results. The $x$ axis means the displacement of finite reflector P. The left $y$ axis means the experimental light intensity received by the receiving fiber $\Psi$ while the right $y$ axis means the simulation light intensity. The plate is moved from $-400 \mu \mathrm{m}$ to $400 \mu \mathrm{m}$.

From Fig. 12 it can be known that the experimental results are in line with the trend of the simulation results. But they are not exactly the same. It is because that the multimode transmitting fiber has the speckle pattern, which doesn't meet the homogeneous Gaussian intensity distribution assumed on the model [10]. Moreover, the surrounding low reflection area of the finite reflective plate, which is assumed to reflect no light, also reflects the light from the transmitting fiber. 


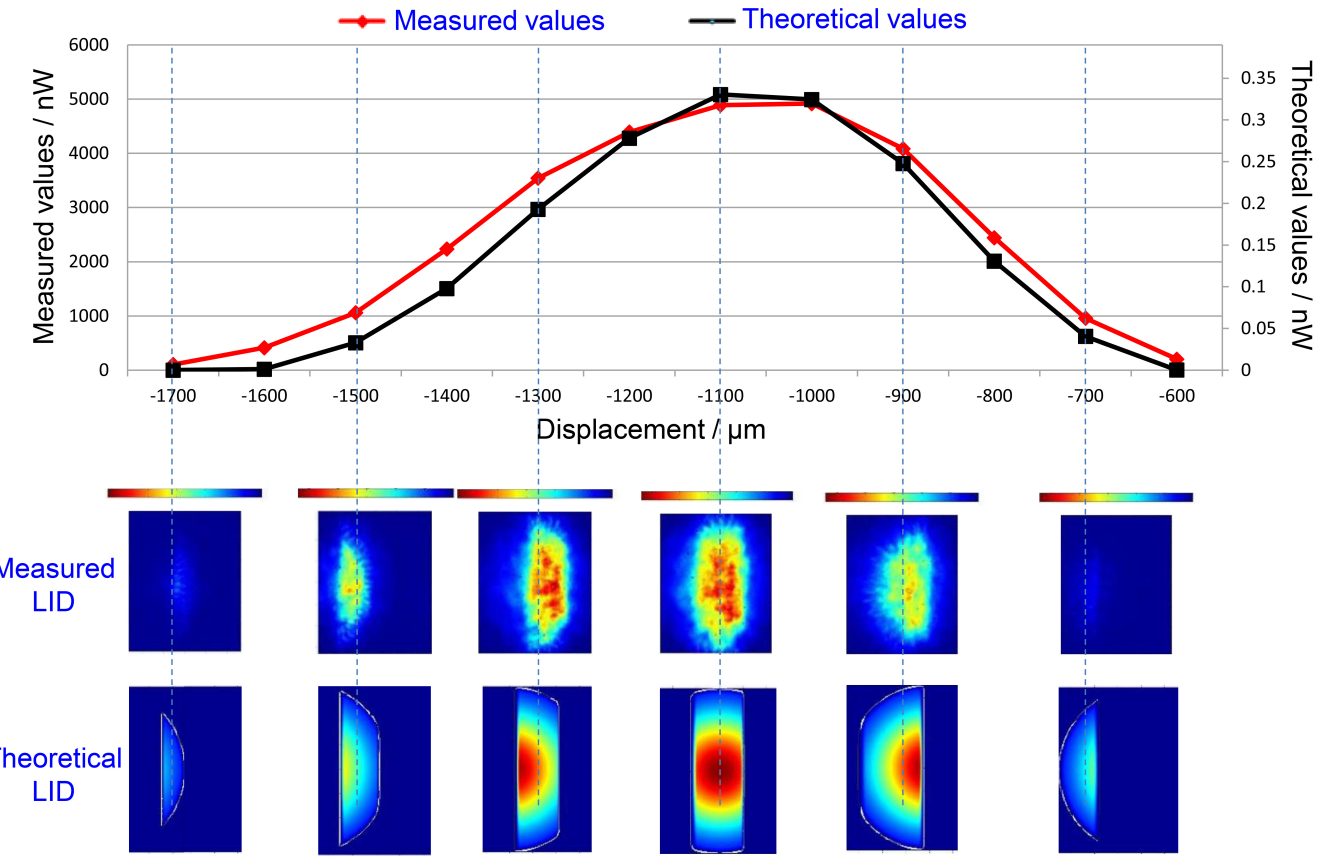

Fig. 11. Theoretical and measured curves.

We can fix an appropriate width of the reflective plate and use the received light intensity curve to measure the displacement. Only left linear scope or right linear scope can be used. During displacement measurement, a certain received light intensity may correspond to several displacements. So we should make sure that which linear scope is used for measurement in advance. For example, the experimental results of $300 \mu \mathrm{m}$ width in Fig. 12 can be used to measure lateral displacement. The left area of the received light intensity curve has a linear scope (for example, from $\mathrm{P}_{1}$ to $\mathrm{P}_{2}$ ). Based on linear fit, we can obtain the gray-colored fitting line $\mathrm{P}_{1} \mathrm{P}_{2}$ and $\mathrm{R}^{2}$ is 0.99999 . Therefore the measuring range of the system is $40 \mu \mathrm{m}$. Due to the resolution of the optical power meter is $10 \mathrm{pW}$ and the slope is $1.332 \mathrm{nW} / \mu \mathrm{m}$, the resolution of the displacement measurement system is about $8 \mathrm{~nm}$.

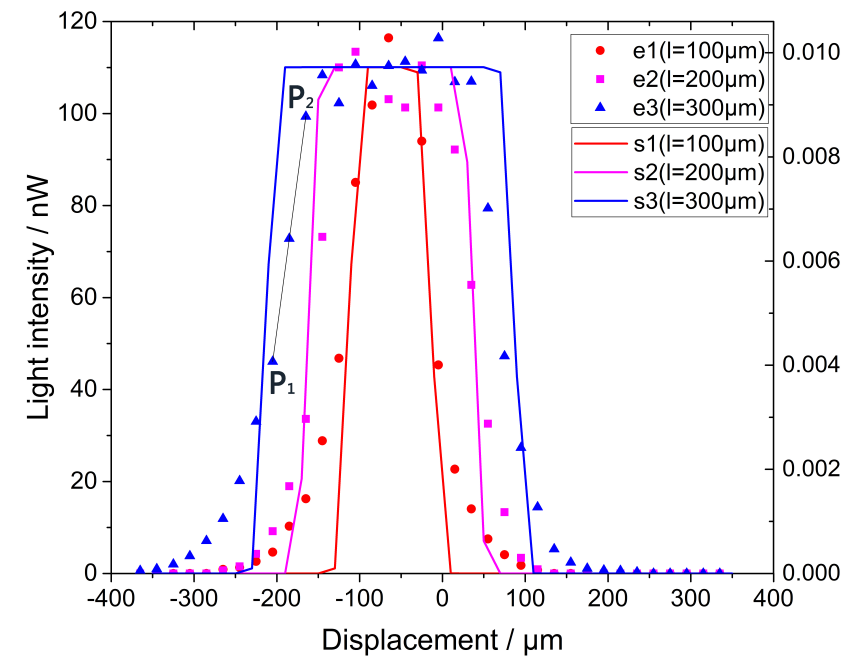

Fig. 12. Relationship between light intensity and displacement.

\subsubsection{Influence of Height $h$}

Change the height of fiber optic probe and a series of experimental curves can be obtained. In these experiments, $R_{r}=25 \mu \mathrm{m}, R_{t}=25 \mu \mathrm{m}$, $l=300 \mu \mathrm{m}, S=125 \mu \mathrm{m}, \alpha=0, \mathrm{NA}=0.22, h$ is changed from $200 \mu \mathrm{m}$ to $1000 \mu \mathrm{m}$ with step $100 \mu \mathrm{m}$.
As shown in Fig. 13, it can be known that there are no essential changes in the peak width with the increase of $h$. The maximum of the received light intensity increase and decrease in turn, which is similar to the simulation analysis.

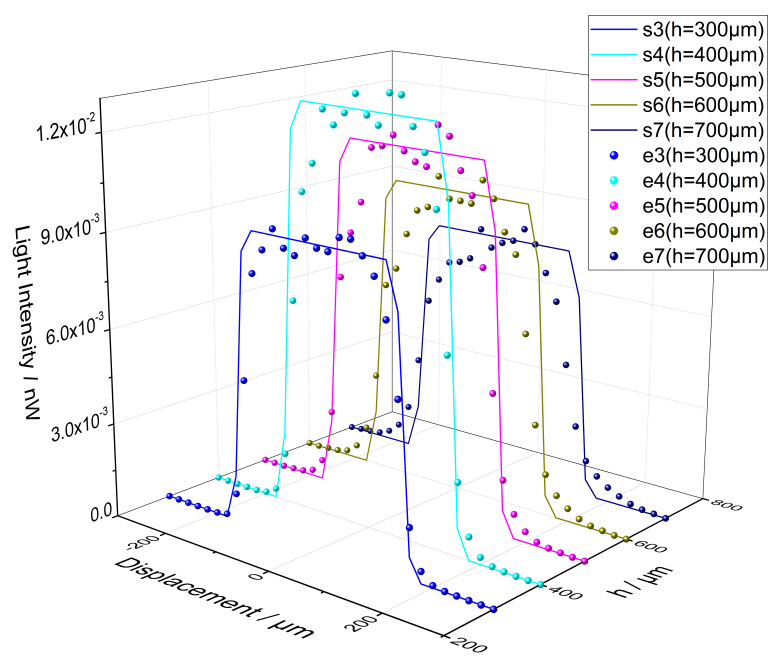

Fig. 13. Relationship between light intensity and displacement.

\section{Conclusion}

The fiber optic displacement measurement model is established on the base of finite reflective surface, and three-dimensional received light intensity distribution is analyzed in both simulation and experiments. Theoretical model is established utilizing rectangular finite reflective plate. Simulation and experiments are carried out to verify the theoretical model. The experimental results show that this measuring model is different from the traditional model. Different parameters such as width and height have great influence on the received light intensity curve. The experiments verify the correctness of the theoretical model with the relative error of light intensity $6.92 \%$.

The displacement measurement model and model based on finite reflector has an enormous range of applications including scientific metrology, precision measurement and optical fiber sensing technology. The proposed model provides a theoretical basis for the 
design of fiber optic displacement measurement sensors, which has an important guiding significance in the practice.

\section{Acknowledgements}

This work is supported by National Science Foundation (NSF) (51275259, 61362035); National Important Scientific Instrument Development Program (2011YQ030134).

\section{References}

[1] F. Restagno, J. Crassous, E. Charlaix, and M. Monchanin, A new capacitive sensor for displacement measurement in a surface-force apparatus. Measurement Science and Technology 12 (2001) 16.

[2] P. Kochert, J. Flugge, D. Miletic, and H.H. Gatzen, Investigation of an advanced micro-inductive sensor, Sensors, 2012 IEEE, IEEE, 2012, pp. 1-4.

[3] B. Chen, X. Cheng, and D. Li, Dual-wavelength interferometric technique with subnanometric resolution. Applied optics 41 (2002) 5933-5937.

[4] B. Zhao, and A. Asundi, Micro-measurement using grating microscopy. Sensors and Actuators A: Physical 80 (2000) 256-264.

[5] H. Golnabi, and P. Azimi, Design and operation of a double-fiber displacement sensor. Optics Communications 281 (2008) 614-620.

[6] M. Shan, R. Min, Z. Zhong, Y. Wang, and Y. Zhang, Differential reflective fiber-optic angular displacement sensor. Optics \& Laser Technology 68 (2015) 124-128.

[7] J.B. Faria, A theoretical analysis of the bifurcated fiber bundle displacement sensor. Instrumentation and Measurement, IEEE Transactions on 47 (1998) 742-747.

[8] L. Yuan, X. Lin, Y. Liang, and Y. Jiang, Applications of angled-mirror in fiber-optic sensors. Optics \& Laser Technology 32 (2000) 255-260.

[9] R. Jafari, and H. Golnabi, Fibre position effects on the operation of optopair fibre displacement sensors. Optics \& Laser Technology 43 (2011) 814819.

[10] J. M. S. Sakamoto, C. Kitano, G. M. Pacheco, and B. R. Tittmann, "High sensitivity fiber optic angular displacement sensor and its application for detection of ultrasound," Appl Optics 51, 4841-4851 (2012).

[11] P.B. Buchade, and A.D. Shaligram, Simulation and experimental studies of inclined two fiber displacement sensor. Sensors and Actuators A: Physical 128 (2006) 312-316.

[12] S.W. Harun, H.Z. Yang, H. Arof, and H. Ahmad, Theoretical and experimental studies on coupler based fiber optic displacement sensor with concave mirror. Optik-International Journal for Light and Electron Optics 123 (2012) 2105-2108.

[13] F. Urban, J. Kadlec, R. Vlach, and R. Kuchta, Design of a pressure sensor based on optical fiber bragg grating lateral deformation. Sensors 10 (2010) 11212-11225.

[14] T. Guo, Q. Zhao, L. Xue, G. Huang, S. Gao, Y. Yan, L. Zhao, and L. Liu, Temperature-independent fiber Bragg grating micro-displacement sensor, Advanced Laser Technologies 2005, International Society for Optics and Photonics, 2006, pp. 634433-634433-7.

[15] M. Bravo, A. Pinto, M. Lopez-Amo, J. Kobelke, and K. Schuster, High precision micro-displacement fiber sensor through a suspended-core Sagnac interferometer. Optics letters 37 (2012) 202-204.

[16] P. Puangmali, H. Liu, K. Althoefer, and L.D. Seneviratne, Optical fiber sensor for soft tissue investigation during minimally invasive surgery. IEEE INTERNATIONAL CONFERENCE ON ROBOTICS AND AUTOMATION (2008) 2934-2939.

[17] Y. Chu, F. Xu, T. Ding, and S. Chen, Measurement of rotation axis transverse displacements based on diffuse reflective intensity modulated optical fiber sensor. OPTICAL ENGINEERING 45 (2006).

[18] A. Zur, and A. Katzir, "Fibers for low-temperature radiometric measurements," Appl Optics 26, 1201-1206 (1987). 